\title{
First-line cetuximab-based chemotherapies for patients with advanced or metastatic $K R A S$ wild-type colorectal cancer
}

\author{
MAMORU UEMURA ${ }^{1,2}$, HO MIN KIM ${ }^{3,4}$, TSUYOSHI HATA ${ }^{2}$, KAZUYA SAKATA ${ }^{5}$, MASAKI OKUYAMA ${ }^{5}$, \\ HIROYOSHI TAKEMOTO ${ }^{6}$, HITOSHI FUJII ${ }^{4}$, TAKAYUKI FUKUZAKI ${ }^{7}$, TETSUSHI MORITA $^{8}$, TAISHI HATA ${ }^{2}$, \\ ICHIRO TAKEMASA $^{2}$, TAROH SATOH ${ }^{2,9}$, TSUNEKAZU MIZUSHIMA ${ }^{2}$, YUICHIRO DOKI ${ }^{2}$ and MASKI MORI ${ }^{2}$, \\ MULTI-CENTER CLINICAL STUDY GROUP OF OSAKA, COLORECTAL CANCER TREATMENT GROUP (MCSGO) \\ ${ }^{1}$ Department of Surgery, National Hospital Organization, Osaka National Hospital, Osaka 540-0006; \\ ${ }^{2}$ Department of Gastroenterological Surgery, Osaka University Graduate School of Medicine, Osaka 565-0871; \\ ${ }^{3}$ Department of Surgery, Osaka Rosai Hospital, Osaka 591-8025; ${ }^{4}$ Department of Surgery, Rinku General Medical Center, \\ Osaka 598-8577; ${ }^{5}$ Department of Surgery, Higashiosaka City General Hospital, Osaka 578-8588; ${ }^{6}$ Department of Surgery, \\ Sakai City Medical Center, Osaka 593-8304; ${ }^{7}$ Department of Surgery, Ikeda Municipal Hospital, Osaka 563-8510; \\ ${ }^{8}$ Department of Surgery, Nissei Hospital, Osaka 550-0012; ${ }^{9}$ Department of Frontier-Science for Cancer \\ and Chemotherapy, Graduate School of Medicine, Osaka 565-0871, Japan
}

Received September 10, 2015; Accepted December 31, 2015

DOI: $10.3892 / \mathrm{mco} .2016 .938$

\begin{abstract}
Colorectal cancer(CRC) is one of the most commonly occurring cancers worldwide. A burgeoning number of studies have demonstrated that the addition of cetuximab to another standard first-line regimen markedly improves the outcome of $\mathrm{CRC}$ treatment. However, at present, the efficacy and safety of cetuximab-based combination chemotherapy has not been well described in Japan. The aim of the present study was to evaluate the efficacy and safety of first-line chemotherapies that included cetuximab for patients with advanced or metastatic Kirsten rat sarcoma viral oncogene homolog (KRAS) wild-type CRC in Japan. This prospective multicenter observational study was conducted at 13 affiliated medical institutions. A total of 64 patients were enrolled between 2010 and 2013. The patients met the following criteria for eligibility: i) histologically confirmed, advanced or metastatic KRAS wild-type CRC; and ii) cetuximab-based chemotherapies administered as a first-line treatment. First-line cetuximab-based treatments were administered as follows: 29 patients (45.3\%) received a combination of infusional fluorouracil, leucovorin and oxaliplatin; 14 patients $(21.9 \%)$ received a combination of capecitabine and oxaliplatin; and 10 patients (15.6\%) received a combination of infusional fluorouracil, leucovorin and irinotecan. The overall response rate (including complete plus
\end{abstract}

Correspondence to: Dr Mamoru Uemura, Department of Surgery, National Hospital Organization, Osaka National Hospital, 2-1-14 Hoenzaka, Chuo-ku, Osaka 540-0006, Japan

E-mail: uemura7777@hotmail.com

Key words: colorectal cancer, cetuximab, oxaliplatin, irinotecan, first-line chemotherapy, KRAS wild-type partial responses) was 50\% (32/64 patients). Initially, 48 lesions were diagnosed as unresectable. Among those, 13 lesions (27.1\%) were converted to a resectable status following cetuximab-based combination chemotherapy treatments. The median overall survival time and the progression-free survival time were 1,189 and 359 days, respectively. The most frequent grade $3 / 4$ adverse event was neutropenia, which occurred in $20.3 \%$ of the patients. The incidence of grade $3 / 4$ skin toxicity was $17.2 \%$ (11/64 patients). Cetuximab-based therapies may represent a promising first-line regimen for patients with advanced or metastatic $K R A S$ wild-type CRC in Japan. In addition, this combination was associated with a low incidence of serious toxicities.

\section{Introduction}

Colorectal cancer (CRC) is one of the most commonly occurring cancers worldwide (1). CRC represents the third largest of the leading causes of cancer-associated mortality in Western countries (2). In recent times there has been a marked increase in the incidence of mortality from CRC in Japan $(3,4)$. At present, cytotoxic agents, including irinotecan, oxaliplatin and fluoropyrimidines, have extended the median survival times (MSTs) of patients with advanced CRC (5-12). Despite these advances, advanced CRC remains incurable in the majority of patients.

Cetuximab is an antibody that specifically inhibits epithelial growth factor receptors. It was initially approved for patients with metastatic CRC lesions who expressed epidermal growth factor receptors, who had failed to respond to irinotecan (topoisomerase inhibitor) therapy (13). The randomized phase III CRYSTAL (cetuximab combined with irinotecan in first-line therapy for metastatic CRC) trial demonstrated the efficacy of first-line treatment with cetuximab plus infusional fluorouracil, leucovorin and irinotecan in patients with metastatic 
CRC tumors lacking mutations in the Kirsten rat sarcoma viral oncogene homolog $(K R A S)$ gene at codons 12 and 13 (hereafter, referred to as $K R A S$ wild-type) (14). In addition, the OPUS (oxaliplatin and cetuximab in first-line treatment of metastatic CRC) study demonstrated that the addition of cetuximab to another standard first-line regimen, fluorouracil, leucovorin and oxaliplatin, markedly improved the response rate and progression-free survival (PFS) time in patients with metastatic KRAS wild-type CRC (15).

Currently, the efficacy and safety of first-line cetuximab-based combination chemotherapies has not been well described in Japan (16). The aim of the present study was to evaluate the efficacy, the safety and the rate of conversion into resectable status of a first-line chemotherapy that included cetuximab in patients with advanced or metastatic KRAS wild-type CRC in Japan.

\section{Materials and methods}

Patient eligibility. This prospective multicenter observational study was conducted at 13 affiliated medical institutions. Institutional review boards at each study site approved the study protocol. A total of 64 patients were enrolled in the study between May 2010 and October 2013. The patients met the following eligibility criteria: i) histologically confirmed advanced or metastatic KRAS wild-type CRC; and ii) cetuximab-based chemotherapies were administered as a first-line treatment.

Study design and assessment. In first-line treatments that included cetuximab, essentially, an initial dose of $400 \mathrm{mg} / \mathrm{m}^{2}$ cetuximab was infused over a $2 \mathrm{~h}$ period, and subsequently, $250 \mathrm{mg} / \mathrm{m}^{2}$ cetuximab was infused over a $1 \mathrm{~h}$ period weekly. Adverse events were graded according to the National Cancer Institute Common Terminology Criteria for Adverse Events, version 4.0. The Response Evaluation Criteria in Solid Tumors (RECIST), version 1.1, was used to evaluate the chemotherapeutic response (17). Responses were defined as either a complete response (CR) or a partial response (PR). The Kaplan-Meier method was used to estimate the PFS and the overall survival (OS) rates, and the log-rank test was used to examine statistical significance. $\mathrm{P}<0.05$ was considered to indicate a statistically significant difference. Statistical analysis was performed with JMP 12 software (SAS Institute, Inc., Cary, NC, USA).

\section{Results}

Patients and treatment. Baseline demographic characteristics are shown in Table I. The median age was 66 years (range: 33-85); 42 patients (65.6\%) were men; and the Eastern Cooperative Oncology Group-Performance Status Scale was 0 in 40 patients $(62.5 \%)$ and 1 in 18 patients $(28.1 \%)$. Overall, 43 patients had metastatic liver lesions; 21 patients had postoperative lesion recurrence; and 43 patients had advanced primary CRC. Among the 21 patients with postoperative recurrences, 15 (71.4\%) were diagnosed with an unresectable lesion. Among the 43 patients with advanced primary CRC, initially, 33 patients $(76.7 \%)$ were diagnosed as unresectable.
Table I. Patient characteristics.

\begin{tabular}{lc}
\hline Characteristic & $\mathrm{n}=64$ (cases) \\
\hline Age (years) & $66(33-85)$ \\
Gender (male/female) & $42 / 22$ \\
PS (0/1/2/3/unknown) & $40 / 18 / 3 / 1$ \\
Disease status ${ }^{\mathrm{a}}$ & \\
Postoperative recurrence & 21 \\
Resectable/unresectable & $6 / 15$ \\
Advanced primary cases & 43 \\
Resectable/unresectable & $10 / 33$ \\
Combined regimens & \\
FOLFOX & 29 \\
FOLFIRI & 11 \\
XELOX & 14 \\
Others & 10 \\
Resection following chemotherapy & \\
Yes & 29 \\
No & 35 \\
\hline
\end{tabular}

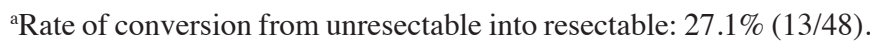
PS, performance status; FOLFOX, infusional fluorouracil, leucovorin and oxaliplatin; FOLFIRI, infusional fluorouracil, leucovorin, and irinotecan; XELOX, capecitabine and oxaliplatin.

Cetuximab was added to first-line treatments, administered as follows: 29 patients $(45.3 \%)$ received a combination of infusional fluorouracil, leucovorin and oxaliplatin; 14 patients (21.9\%) received a combination of capecitabine and oxaliplatin; and 10 patients $(15.6 \%)$ received a combination of infusional fluorouracil, leucovorin and irinotecan. The median follow-up period following the beginning of treatment was 1,093 days (range: 385-1,592). The median duration of the first-line cetuximab-based treatment was 14 weeks (range: 2-87).

Efficacy. The efficacy of the cetuximab-based treatment is summarized in Table II. The overall response rate (CR plus PR) was 50\% (32/64 patients). Confirmed CRs or PRs occurred in 29 patients $(63.1 \%)$ who received oxaliplatin-based treatment, and in three patients $(25.0 \%)$ who received irinotecan-based treatment. Initially, 48 patients were diagnosed with unresectable lesions. Among these, 13 lesions (27.1\%) were converted to a resectable status following treatment with a cetuximab-based combination chemotherapy. Among these 13 patients with converted lesions, 10 had been initially diagnosed with non-resectable liver metastases. The conversion rate was higher in the oxaliplatin-based group $(12 / 30 ; 40.0 \%)$ compared with the irinotecan-based group $(1 / 12 ; 8.3 \%)$.

The MST was 1,189 days. Patients who had received surgical resections following treatment $(n=29)$ had a significantly longer MST compared with patients with surgically unresectable lesions (MST: 1,339 and 806 days, respectively; $\mathrm{P}=0.0006$; Fig 1A). In patients with initially unresectable lesions $(n=48)$, the MST was 872 days. In this subgroup, patients with tumors that converted to a surgical resect- 
Table II. Efficacy of cetuximab added to different combination treatments.

\begin{tabular}{|c|c|c|c|}
\hline \multirow[b]{2}{*}{ Response rate } & \multicolumn{2}{|c|}{ Patients } & \multirow[b]{2}{*}{$\mathrm{CR}+\mathrm{PR}(\%)$} \\
\hline & No. & Percentage of total $(\%)$ & \\
\hline \multicolumn{4}{|c|}{ Total cases $(n=64)$} \\
\hline $\mathrm{CR}$ & 1 & 1.6 & 50.0 \\
\hline PR & 31 & 48.4 & \\
\hline SD & 20 & 31.2 & \\
\hline PD & 8 & 12.5 & \\
\hline NE & 4 & 6.2 & \\
\hline \multicolumn{4}{|c|}{ Oxaliplatin-based $(n=46)$} \\
\hline $\mathrm{CR}$ & 1 & 2.2 & 63.1 \\
\hline PR & 28 & 60.9 & \\
\hline SD & 14 & 30.4 & \\
\hline PD & 1 & 2.2 & \\
\hline $\mathrm{NE}$ & 2 & 4.3 & \\
\hline \multicolumn{4}{|c|}{ Irinotecan-based $(\mathrm{n}=12)$} \\
\hline $\mathrm{CR}$ & 0 & 0.0 & 25.0 \\
\hline PR & 3 & 25.0 & \\
\hline SD & 3 & 25.0 & \\
\hline PD & 6 & 50.0 & \\
\hline NE & 0 & 0.0 & \\
\hline \multicolumn{4}{|l|}{ Others $(n=6)$} \\
\hline $\mathrm{CR}$ & 0 & 0.0 & 0.0 \\
\hline PR & 0 & 0.0 & \\
\hline SD & 3 & 50.0 & \\
\hline PD & 1 & 16.7 & \\
\hline NE & 2 & 33.3 & \\
\hline
\end{tabular}

CR, complete response; PR, partial response; $\mathrm{SD}$, stable disease; $\mathrm{PD}$, progressive disease; NE, not evaluable.
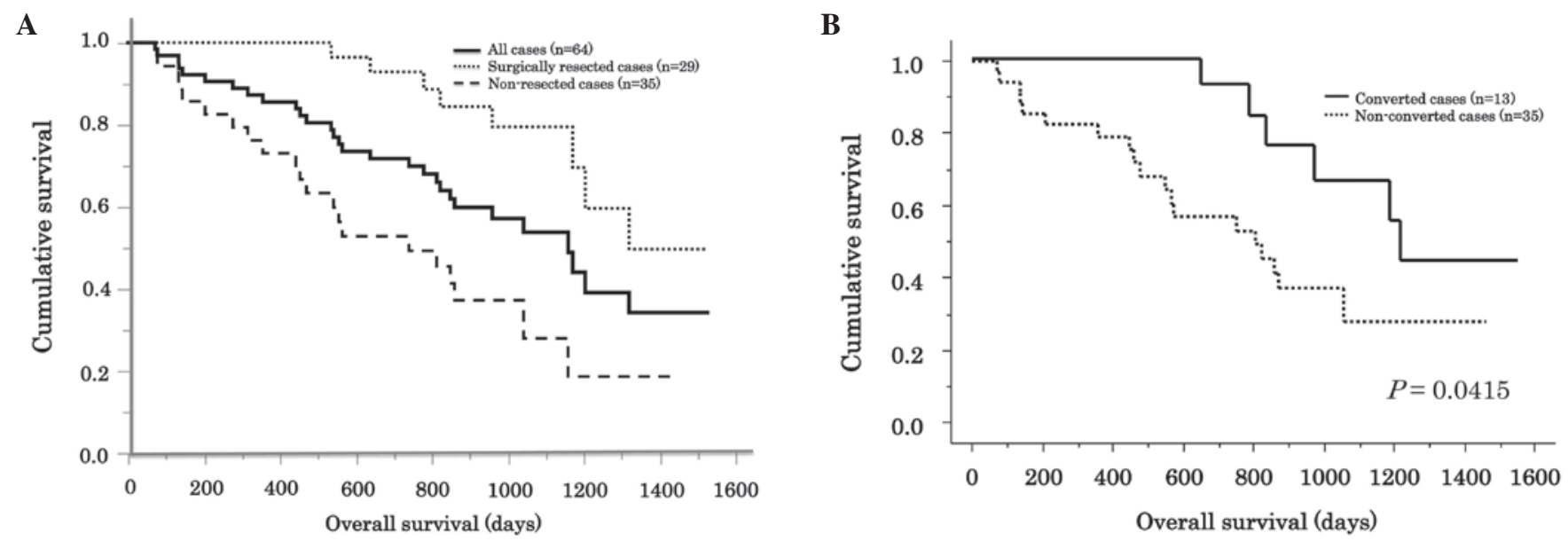

Figure 1. Kaplan-Meier curves indicating the overall survival rates. The curves show (A) all patients $(\mathrm{n}=64)$, and (B) patients with initially non-resectable lesions.

able status had a significantly longer MST compared with patients with unresectable lesions (MST: 1,551 and 806 days, respectively; $\mathrm{P}=0.0415$; Fig 1B). The overall median PFS time was 359 days. Patients who received surgical resections following treatment $(n=29)$ had a significantly longer median PFS time (432 days) compared with patients with surgically unresectable lesions ( $n=35$; median PFS time, 196 days; $\mathrm{P}=0.0056$; Fig 2). 
Table III. Adverse events.

\begin{tabular}{|c|c|c|c|c|c|c|}
\hline \multirow[b]{2}{*}{ Adverse event } & \multicolumn{2}{|c|}{ Grade $1-2$} & \multicolumn{2}{|c|}{ Grade 3-4 } & \multicolumn{2}{|c|}{ Total } \\
\hline & $\mathrm{n}$ & $\%$ & $\mathrm{n}$ & $\%$ & $\mathrm{n}$ & $\%$ \\
\hline \multicolumn{7}{|l|}{ Skin toxicity } \\
\hline All (maximum toxicity) & 28 & $(43.8)$ & 11 & $(17.2)$ & 39 & $(60.9)$ \\
\hline Acneiform eruption & 28 & $(43.8)$ & 7 & $(10.9)$ & 35 & $(54.7)$ \\
\hline Rhagades & 18 & $(28.1)$ & 4 & $(6.2)$ & 22 & $(34.3)$ \\
\hline Peronychia & 23 & $(35.9)$ & 2 & $(3.1)$ & 25 & $(39.0)$ \\
\hline \multicolumn{7}{|l|}{ Hematological toxicity } \\
\hline Neutropenia & 12 & (18.9) & 13 & $(20.3)$ & 25 & $(39.0)$ \\
\hline Leukopenia & 12 & $(18.9)$ & 12 & $(18.9)$ & 24 & $(37.8)$ \\
\hline Thrombocytopenia & 13 & $(20.3)$ & 2 & $(3.1)$ & 15 & $(23.4)$ \\
\hline Anemia & 6 & (9.4) & 0 & $(0.0)$ & 6 & $(9.4)$ \\
\hline \multicolumn{7}{|l|}{ Others } \\
\hline Peripheral neuropathy & 24 & (37.5) & 3 & (4.7) & 27 & $(42.2)$ \\
\hline Fatigue & 16 & $(25.0)$ & 2 & (3.1) & 18 & $(28.1)$ \\
\hline Anorexia & 11 & $(17.2)$ & 1 & (1.6) & 12 & $(18.9)$ \\
\hline Stomatitis & 8 & $(12.5)$ & 2 & $(3.1)$ & 10 & $(15.6)$ \\
\hline Nausea, vomiting & 5 & $(7.8)$ & 1 & $(1.6)$ & 6 & (9.4) \\
\hline
\end{tabular}

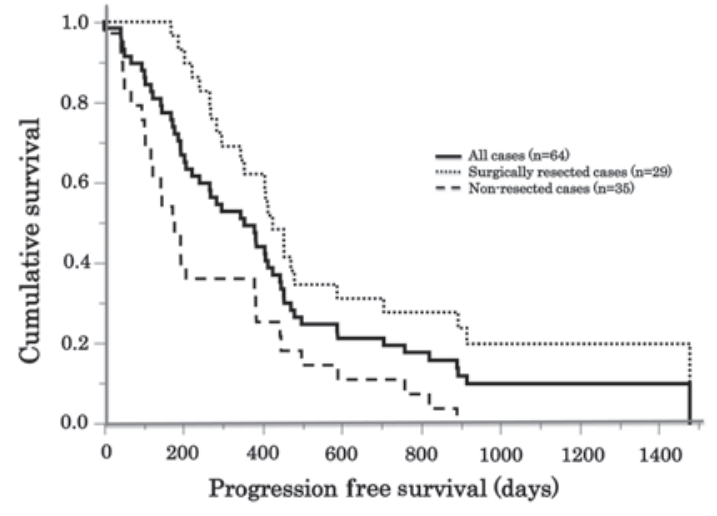

Figure 2. Kaplan-Meier curve for progression free survival. All patients enrolled for the present study are included $(n=64)$.

Adverse events. Adverse events were graded for all treatment groups (Table III). No treatment-associated mortalities were identified during the therapy. The most frequent grade $3 / 4$ adverse event was neutropenia, which occurred in $20.3 \%$ of the patients. The incidence of grade $3 / 4$ skin toxicity was $17.2 \%$ (11/64 patients). Among skin toxicities, acneiform eruption was the most common (7/64 patients; 10.9\%). Grade 4 skin toxicities were not observed in the present study.

\section{Discussion}

In the present study, it was identified that, among patients with advanced or metastatic KRAS wild-type CRC, one-half (50\%) responded well to cetuximab-based therapies, whereas approximately one-third (27\%) of unresectable lesions were converted to resectable status. These findings were consistent with results obtained from the CRYSTAL trial, which revealed that the addition of cetuximab to a first-line regimen improved the clinical outcome in patients with advanced KRAS wild-type CRC (14). In another study, the vascular endothelial growth factor-A antibody, bevacizumab, combined with a first-line regimen was demonstrated to be effective for patients with advanced CRC in a phase III trial (18). However, results obtained from the FIRE-3 trial suggested that cetuximab may be preferable to bevacizumab in combination therapies as a first-line regimen for patients with $K R A S$ wild-type tumors (19).

In the CRYSTAL trial, the median PFS time was 9.9 months, and the median OS time was 24.9 months for patients with KRAS wild-type CRC who received a cetuximab-based treatment (14). Similarly, in the OPUS trial, the median PFS time was 8.3 months, and the median OS time was 22.8 months for patients with $K R A S$ wild-type CRC who received a cetuximab-based treatment (15). In the current study, the median PFS time was 11.0 months, and the median OS time was 39.6 months. Additionally, patients who received oxaliplatin-based treatments had a median PFS time of 14.4 months and a median OS time of 44.6 months. However, due to the relatively small number of patients who received oxaliplatin-based treatment, these outcomes may have been overestimated. Nevertheless, our results have revealed treatment efficacy comparable with previous reports.

In the present study, 29 patients received surgical resections following first-line cetuximab-based combination chemotherapies. The rate of true conversion from non-resectable to resectable status was identified to be $27.1 \%$ (13/48 patients). This finding was consistent with results from a previous phase II trial, which assessed cetuximab in a neoadjuvant treatment for non-resectable colorectal liver metastases (the CELIM trial) (20). In that trial, the true conversion rate from non-resectable to resectable lesions was $28.0 \%$ (19/68 patients). 
In the present study, 31 patients initially had non-resectable liver metastases. Among these, 10 patients became resectable following first-line cetuximab combined therapies. Therefore, in a setting similar to that of the CELIM trial, the conversion rate in the present study was $32.2 \%$ (10/31 patients). These changes in resectability also provided satisfactory treatment efficacy, comparable with those observed previously (20).

Adverse events, particularly skin reactions, are important factors that influence treatment compliance for cetuximabbased treatments. The incidence of grade 3/4 skin reactions was reported to be $18-40 \%$ in previous studies $(13-15,19,20)$. In the present study, the incidence of grade $3 / 4$ skin toxicity was $17.2 \%$. Notably, no grade 4 skin toxicity was observed. These results suggested that adverse events associated with a first-line cetuximab-based treatment were limited, and comparable with those described in previous reports.

In conclusion, the present study has demonstrated that cetuximab-based therapies represent a promising first-line regimen for advanced or metastatic $K R A S$ wild-type CRC in Japan. Additionally, a low incidence of serious toxicities was observed.

\section{References}

1. Parkin DM, Bray F, Ferlay J and Pisani P: Global cancer statistics, 2002. CA Cancer J Clin 55: 74-108, 2005.

2. Jemal A, Siegel R, Xu J and Ward E: Cancer statistics, 2010. CA Cancer J Clin 60: 277-300, 2010.

3. Center MM, Jemal A and Ward E: International trends in colorectal cancer incidence rates. Cancer Epidemiol Biomarkers Prev 18: 1688-1694, 2009.

4. Colorectal Cancer Estimated Incidence Mortality and Prevalence Worldwide in 2012. http://globocan.iarc.fr/Pages/fact_sheets_ cancer.aspx.

5. Cassidy J, Twelves C, Van Cutsem E, Hoff P, Bajetta E, Boyer M, Bugat R, Burger U, Garin A, Graeven U, et al: First-line oral capecitabine therapy in metastatic colorectal cancer: A favorable safety profile compared with intravenous 5-fluorouracil/leucovorin. Ann Oncol 13: 566-575, 2002.

6. Cunningham D, Pyrhönen S, James RD, Punt CJ, Hickish TF, Heikkila R, Johannesen TB, Starkhammar H, Topham CA, Awad L, et al: Randomised trial of irinotecan plus supportive care versus supportive care alone after fluorouracil failure for patients with metastatic colorectal cancer. Lancet 352: 1413-1418, 1998.

7. Rougier P, Van Cutsem E, Bajetta E, Niederle N, Possinger K, Labianca R, Navarro M, Morant R, Bleiberg H, Wils J, et al: Randomised trial of irinotecan versus fluorouracil by continuous infusion after fluorouracil failure in patients with metastatic colorectal cancer. Lancet 352: 1407-1412, 1998.

8. Saltz LB, Cox JV, Blanke C, Rosen LS, Fehrenbacher L, Moore MJ, Maroun JA, Ackland SP, Locker PK, Pirotta N, et al: Irinotecan plus fluorouracil and leucovorin for metastatic colorectal cancer. Irinotecan study group. N Engl J Med 343: 905-914, 2000.
9. Douillard JY, Cunningham D, Roth AD, Navarro M, James RD, Karasek P, Jandik P, Iveson T, Carmichael J, Alakl M, et al: Irinotecan combined with fluorouracil compared with fluorouracil alone as first-line treatment for metastatic colorectal cancer: A multicentre randomised trial. Lancet 355: 1041-1047, 2000.

10. Goldberg RM, Sargent DJ, Morton RF, Fuchs CS, Ramanathan RK, Williamson SK, Findlay BP, Pitot HC and Alberts SR: A randomized controlled trial of fluorouracil plus leucovorin, irinotecan and oxaliplatin combinations in patients with previously untreated metastatic colorectal cancer. J Clin Oncol 22: 23-30, 2004.

11. Grothey A, Sargent D, Goldberg RM and Schmoll HJ: Survival of patients with advanced colorectal cancer improves with the availability of fluorouracil-leucovorin, irinotecan and oxaliplatin in the course of treatment. J Clin Oncol 22: 1209-1214, 2004.

12. Uehara $\mathrm{K}$ and Nagino M: Neoadjuvant treatment for locally advanced rectal cancer: A systematic review. Surg Today, 2015 (Epub ahead of print).

13. Cunningham D, Humblet Y, Siena S, Khayat D, Bleiberg H, Santoro A, Bets D, Mueser M, Harstrick A, Verslype C, et al: Cetuximab monotherapy and cetuximab plus irinotecan in irinotecan-refractory metastatic colorectal cancer. N Engl J Med 351: 337-345, 2004.

14. Van Cutsem E, Köhne CH, Hitre E, Zaluski J, Chang Chien CR, Makhson A, D'Haens G, Pintér T, Lim R, Bodoky G, et al: Cetuximab and chemotherapy as initial treatment for metastatic colorectal cancer. N Engl J Med 360: 1408-1417, 2009.

15. Bokemeyer C, Bondarenko I, Makhson A, Hartmann JT, Aparicio J, de Braud F, Donea S, Ludwig H, Schuch G, Stroh C, et al: Fluorouracil, leucovorin and oxaliplatin with and without cetuximab in the first-line treatment of metastatic colorectal cancer. J Clin Oncol 27: 663-671, 2009.

16. Saeki H, Emi Y, Kumashiro R, Otsu H, Kawano H, Ando K, Ida S, Kimura Y, Tokunaga E, Oki E, et al: Impact of second-line and later cetuximab-containing therapy and KRAS genotypes in patients with metastatic colorectal cancer: A multicenter study in Japan. Surg Today 44: 1457-1464, 2014.

17. Eisenhauer EA, Therasse P, Bogaerts J, Schwartz LH, Sargent D, Ford R, Dancey J, Arbuck S, Gwyther S, Mooney M, et al: New response evaluation criteria in solid tumours: Revised RECIST guideline (version 1.1). Eur J Cancer 45: 228-247, 2009.

18. Hurwitz H, Fehrenbacher L, Novotny W, Cartwright T, Hainsworth J, Heim W, Berlin J, Baron A, Griffing S, Holmgren E, et al: Bevacizumab plus irinotecan, fluorouracil and leucovorin for metastatic colorectal cancer. N Engl J Med 350: 2335-2342, 2004.

19. Heinemann V, von Weikersthal LF, Decker T, Kiani A, Vehling-Kaiser U, Al-Batran SE, Heintges T, Lerchenmüller C, Kahl C, Seipelt G, et al: FOLFIRI plus cetuximab versus FOLFIRI plus bevacizumab as first-line treatment for patients with metastatic colorectal cancer (FIRE-3): A randomised, open-label, phase 3 trial. Lancet Oncol 15: 1065-1075, 2014.

20. Folprecht G, Gruenberger T, Bechstein WO, Raab HR, Lordick F, Hartmann JT, Lang H, Frilling A, Stoehlmacher J, Weitz J, et al: Tumour response and secondary resectability of colorectal liver metastases following neoadjuvant chemotherapy with cetuximab: The CELIM randomised phase 2 trial. Lancet Oncol 11: 38-47, 2010. 